\title{
Reach Improvement of Mode Division Multiplexed Systems using Fiber Splices
}

\author{
Filipe Ferreira, Student Member, IEEE, Daniel Fonseca, Adriana Lobato, \\ Beril Inan, Student Member, IEEE, and Henrique Silva, Member, IEEE
}

\begin{abstract}
This letter proposes the introduction of discrete modal crosstalk (XT) through fiber splices for the improvement of the distance reach (DR) of mode division multiplexed (MDM) transmission systems over few mode fibers (FMFs). The proposed method increases the DR reducing the time spread of the FMFs impulse response. The effectiveness of this method is assessed through simulation considering $3 \times 136 \mathrm{Gbit} / \mathrm{s}$ MDM-coherentlydetected polarization-multiplexed quadrature-phase-shift-keying ultralong-haul transmission systems employing inherently low differential mode delay (DMD) FMFs or DMD compensated FMFs. A maximum DR increase factor of 1.9 is obtained for the optimum number of splices per span and splice XT level.
\end{abstract}

Index Terms-Few-Mode Fibers, Differential Mode Delay, Modal Crosstalk, Mode Dependent Losses.

\section{INTRODUCTION}

$\mathrm{M}$ ODE-Division MultipleXING (MDM) over few-mode fibers (FMFs) has been proposed as a next-generation solution to overcome the impeding installed capacity exhaustion of current single-mode fibers (SMFs) [1]. However, the equalizer complexity is significantly higher than that required for SMFs due to the channel impulse response (CIR) spread over time, resulting from the combined effect of differential mode delay (DMD) and modal crosstalk (XT) [1]. Recently, the minimization of the CIR spread along FMFs has been investigated considering random unitary crosstalk

Manuscript received February 21, 2013; revised March 28, 2013; accepted March 28, 2013. Date of publication April 2, 2013. Manuscript received January 18, 2013. This work has been partially supported by: Nokia Siemens Networks, Fundação para a Ciência e Tecnologia (FCT) under the grant SFRH/BDE/51094/2010, and European Communities $7^{\text {th }}$ Framework Programme under grant agreement 228033 (MODE-GAP).

F. Ferreira is with Nokia Siemens Networks Portugal, S. A., Amadora 2720-093, Portugal, and also with Instituto de Telecomunicações, Universidade de Coimbra, Coimbra 3030-290, Portugal (e-mail: filipe.ferreira@nsn.com).

D. Fonseca is with Nokia Siemens Networks Portugal, S. A., Amadora 2720-093, Portugal, and also with Instituto de Telecomunicações, Lisboa 1049-001, Portugal (e-mail: daniel.fonseca@nsn.com). A. Lobato is with the University of Federal Armed Forces, Munich 85579, Germany (e-mail: adriana.lobato@unibw.de).

B. Inan is with the Institute of Communications Engineering, Technische Universität München, Munich 81675, Germany (e-mail: beril.inan@tum.de).

H. Silva is with Instituto de Telecomunicações, Universidade de Coimbra, Coimbra 3030-290, Portugal (e-mail: hjas@co.it.pt).

Color versions of one or more of the figures in this letter are available online at http://ieeexplore.ieee.org.

Digital Object Identifier 10.1109/LPT.2013.2256120 matrices distributed along the FMFs [2]-[3]. However, given that current experimental FMFs present weak distributed XT (dXT) (reported value of $-18 \mathrm{~dB}$ after $33 \mathrm{~km} \mathrm{[1],} \mathrm{[4]),} \mathrm{the} \mathrm{XT}$ accumulated along transmission can be increased using discrete XT, introduced at specific points. In [5] it has been shown that ideal discrete mode permutation, without mode dependent losses (MDL), within the transmission line may effectively increase the maximum distance reach by a factor of 5. However, such conclusion was based on the statistics of multiple-input-multiple-output (MIMO) outage capacity, ignoring DMD and optical signal-to-noise ratio (OSNR).

In this letter, we propose the introduction of displaced core fiber splices along the transmission fiber as a practical method to implement discrete mode permutation. As such splices introduce MDL, the transmission distance gain is evaluated considering ultralong-haul transmission simulations along inherently low DMD FMFs (ILD-FMFs) and DMD compensated FMFs (DC-FMFs), supporting 3 LP modes (LP01, LP11a and LP11b). In Section II, the different sources of XT and MDL considered in this letter and respective models are presented. Additionally, Section II provides a description of the simulation setup and a conceptual discussion about the principle behind the use of fiber splices for reach improvement. Section III presents simulation results showing a distance increase by a maximum factor of 1.9 . Conclusions are drawn in Section IV.

\section{CONCEPT Discussion AND SiMUlATION SETUP}

\section{A. Modeling of modal XT and MDL}

There are several sources of modal XT and MDL when considering transmission along FMFs: waveguide imperfections distributed along the fiber, fiber bends and fiber splices. The XT introduced by the waveguide imperfections and the fiber bends can be described as distributed XT (dXT) along the FMF, and the splice XT (sXT) can be considered as discrete XT introduced at a specific point of the link. Along this paper, modal XT (sXT or accumulated dXT) is calculated as $\mathrm{XT}=\left(P_{L P 11 a}+P_{L P 11 b}\right) / P_{L P 01}$, where $P_{L P 11 a}, P_{L P 11 b}$ and $P_{L P 01}$ are the powers of modes LP11a, LP11b and LP01, respectively, after the fiber segment (or splice) under test, when only the LP01 was launched. The dXT is modeled using the work presented in [6], where the fiber is divided into multiple sections each with a random displacement of the core center position. Considering a fiber step of $200 \mathrm{~m}$, the core displacement distribution parameters have been tuned in order 


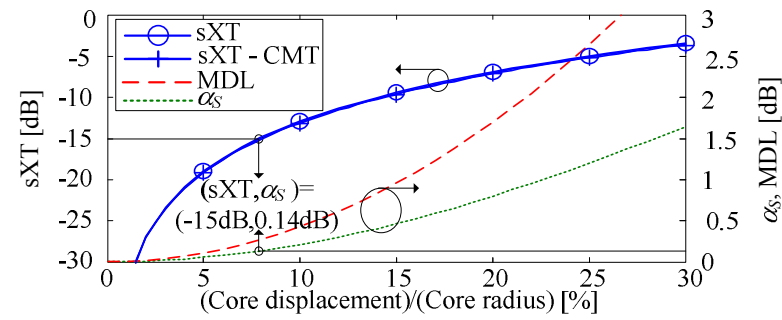

Fig. 1. Splice XT, $\alpha_{S}$, and MDL as a function of the core displacement.

to match the experimental measurements presented in [1], where the accumulated $\mathrm{dXT}$ at the end of $33 \mathrm{~km}$ was $-18 \mathrm{~dB}$. Additionally, MDL arising from the waveguide imperfections and bends is neglected according to the experimental measurements presented in [1], [4].

Regarding splices, the displacement of the fiber core breaks the orthogonality between the field spatial distributions of the modes, introducing sXT and MDL. Along this paper, the transfer matrix of the splices has been computed through an overlap integral of the field distribution over the fiber cross section, except when it is said explicitly that the MDL is neglected. For those cases, the transfer matrix is calculated using the coupled-mode theory (CMT) considering only the guided-modes. The splice loss $\left(\alpha_{S}\right)$ and the MDL are computed as described in [7]. Fig. 1 shows the sXT, $\alpha_{S}$ and MDL as functions of the radial displacement. Note that the difference between the sXT given by the overlap integral and by the CMT can be considered negligible. The refractive index profile considered to obtain the results in Fig. 1 (and along this letter) is composed by a graded core and a cladding trench, since this profile proved to be capable of guaranteeing arbitrary DMD [4], [8]. The results in Fig. 1 are for the case $\mathrm{DMD}=0 \mathrm{ps} / \mathrm{km}$. The variation of the sXT, MDL and $\alpha_{\mathrm{s}}$ dependencies on displacement among the FMFs considered in this letter is almost negligible.

In practical cases, the target sXT intended for each splice can be set during splicing. This can be achieved using state of the art fusion splicer machines, capable of introducing a fixed loss with a $0.01 \mathrm{~dB}$ step (for SMFs) through an intentional core axial offset, using a $0.01 \mu \mathrm{m}$ stepper motor (less than $0.1 \%$ of displacement in Fig. 1). Assuming such accuracy, variation of sXT in steps of $0.5 \mathrm{~dB}$ above $-25 \mathrm{~dB}$ is expected.

\section{B. Simulation Setup}

The simulation setup is shown in Fig. 2. The transmitter is composed by 3 single mode transmitters (SMTX) generating 3 signals at the same wavelength carrying $136 \mathrm{Gbit} / \mathrm{s}$ each, from which $24 \%$ are forward error correction and $10 \%$ are training symbols, using coherently detected polarization multiplexed quadrature-phase-shift-keying (CP-QPSK) modulation modeled as in [9]. The 3 data signals are launched into the FMF using a mode multiplexer (MMUX). In the MMUX, each input corresponds to a different launch mode: LP01, LP11a and LP11b. The mode demultiplexer (MDMUX) performs the reciprocal function. The MMUXs/MDMUXs are modeled by an insertion loss of $5.2 \mathrm{~dB}$ per mode [10].

The fiber span has a total length $(L)$ of $80 \mathrm{~km}$ composed by $m$ segments of equal length $(L s)$, each segment with $2 \times m-1$

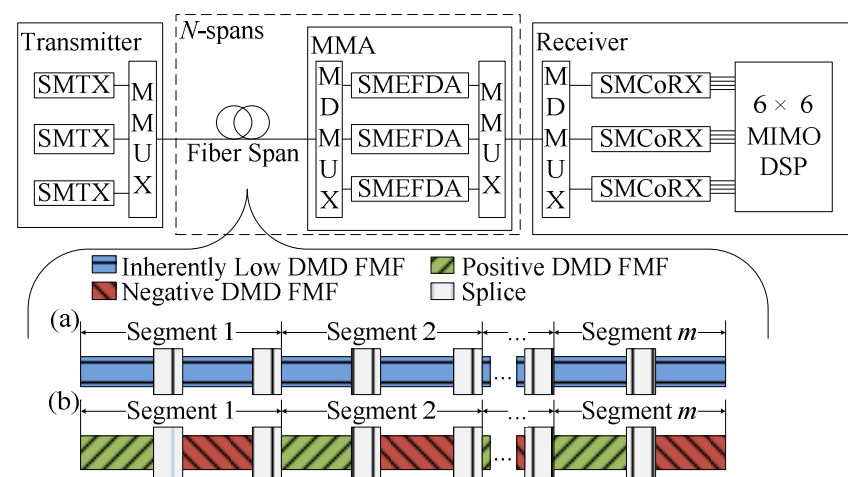

Fig. 2. Span configuration: (a) ILD-FMF link and (b) DC-FMF link.

splices. Two different configurations are considered to achieve low DMD transmission: ILD-FMFs [4], Fig. 2 a), and DC-FMFs [11], Fig. 2 b). An ILD- FMF segment is composed by two pieces of FMF of equal length and DMD $=l D M D$. A DC-FMF segment is composed by one positive DMD FMF with $\mathrm{DMD}=p D M D$ and length $L p$ and one negative DMD FMF with DMD $=n D M D$ and length $L n$. The average DMD $(a D M D)$ for the last segment type is $a D M D=[p D M D \times L p+$ $n D M D \times L n] / L s$. The simulation considered $p D M D$ equal to $200 \mathrm{ps} / \mathrm{km}, n D M D$ equal to $-200 \mathrm{ps} / \mathrm{km}, l D M D$ and $a D M D$ ranging from 0 to $9 \mathrm{ps} / \mathrm{km}$ (according to the experimental measurements presented in [4], [11]). The range for $l D M D$ and $a D M D$ takes into account practical cases of wavelengthdivision-multiplexed signals where the DMD slope is nonnegligible. The $a D M D$ was set changing $L^{+} / L^{-}$. The $I D M D$, $p D M D$ and $n D M D$ values of the respective FMFs were set through the optimization of the refractive index profile, as presented in [8]. The refractive index profile was optimized for an effective core area of the LP01 mode of $\sim 200 \mu \mathrm{m}^{2}$, in order to guarantee that linear transmission along the FMF can be assumed with $5 \mathrm{dBm}$ of launch power per mode [6]. Regarding the fiber loss, the value considered was $0.19 \mathrm{~dB} / \mathrm{km}$, independently of the mode considered, as experimentally verified in [1], [4]. The polarization mode dispersion, dispersion and dispersion slope values are $1 \mathrm{ps} / \sqrt{\mathrm{km}}, 22 \mathrm{ps} / \mathrm{km} / \mathrm{nm}$ and $0.06 \mathrm{ps} /\left(\mathrm{nm}^{2} \cdot \mathrm{km}\right)$, respectively, independently of the LP mode.

The multimode amplifier (MMA) is composed by 3 single mode erbium doped amplifiers (SMEDFAs) arranged between a MMUX and a MDEMUX, as experimentally implemented in [12]. The SMEDFAs gains are set to guarantee that the launch power per mode at the output of the MMA is $5 \mathrm{dBm}$. Note that this amplifier allows the correction of the accumulated MDL. The SMEDFAs have a $5 \mathrm{~dB}$ noise figure (NF).

The receiver is composed by a MDMUX followed by 3 typical single mode coherent receiver front-ends employing polarization diversity (SMCoRXs). The outputs of the SMCoRXs are then connected to a DSP to mitigate transmission effects, namely dispersion, XT, and DMD between modes and polarizations. A $6 \times 6$ MIMO DSP is used, since FMFs with 3 LP modes (each with 2 polarizations) are considered. The DSP used is the $6 \times 6$ data-based equalizer presented in [9], considering 1001 taps, allowing mitigation of the transmission effects contained within a time interval of $15 \mathrm{~ns}$. 


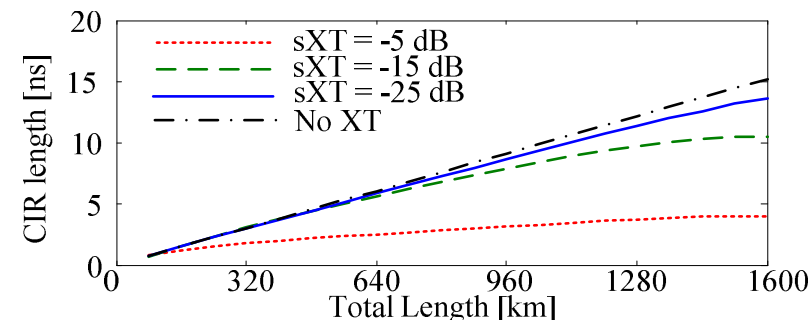

(a)
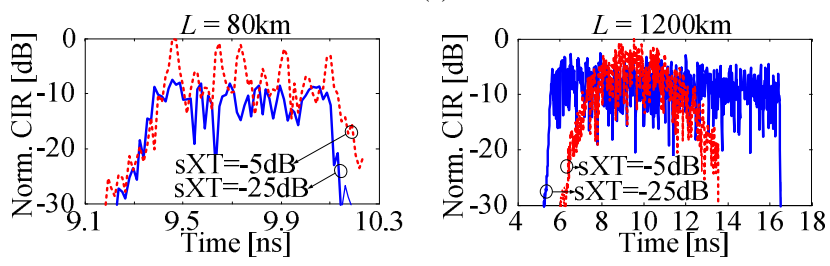

(b)

Fig. 3. (a) CIR length as a function of fiber length, for $m=4$ without splice MDL. (b) Normalized CIR for $L=80 \mathrm{~km}$ and $1200 \mathrm{~km}$.

The performance was measured considering: the required OSNR (rONSR) for a $10^{-2}$ bit-error-ratio on the worst mode, and the available OSNR (aOSNR) on the worst mode (determined by the launch power, the SMEDFAs NF, the fiber losses, the MMUX/MDMUX losses, and the splice MDL).

\section{Conceptual Discussion}

This sub-section explains the principle behind the use of fiber splices for reach improvement, by analyzing the CIR time spread due to the combined effect of DMD and XT.

The CIR provides a qualitative evaluation of the required equalizer complexity, particularly the time length of the CIR, as the number of taps required to compensate correctly the transmission effects scales with the CIR length. The CIR is calculated using a least square error estimator, obtaining the 36 impulse responses of the $6 \times 6$ MIMO channel. Afterwards, the CIR length is computed as the time interval containing $95 \%$ of the power of the sum of the 36 impulse responses (less than $100 \%$ in order to exclude the CIR points with very low amplitude, and thus negligible impact in the equalization) In order to evaluate the dependence of the CIR length on the transmission distance, Fig. 3(a) presents the CIR length for the span configuration in Fig. 2(a), with: $l D M D=9 \mathrm{ps} / \mathrm{km}, m=4$ and different levels of sXT introduced by each splice, neglecting MDL. The purpose of neglecting the MDL at this point is to separate the two degrading effects discussed along this letter: the MDL and the residual crosstalk due to a limited MIMO receiver memory. Fig. 3(a) shows that the CIR length scales almost linearly with transmission distance for $\mathrm{sXT}=-25 \mathrm{~dB}$ (consistent with the weak $\mathrm{dXT}$ ), as can be concluded by comparing with the dashed line representing the CIR length in the absence of any form of XT. Increasing sXT, Fig. 3(a) shows that a higher transmission distance can be reached for the same CIR length, and thus for the same equalization complexity. To better understand this behavior, Fig. 3(b) shows the CIR for $80 \mathrm{~km}$ and $1200 \mathrm{~km}$. For $80 \mathrm{~km}$, the CIR length is almost the same for sXT equal to $-25 \mathrm{~dB}$ and $-5 \mathrm{~dB}$. However, for $1200 \mathrm{~km}$, the CIR is clearly compressed for $\mathrm{sXT}=-5 \mathrm{~dB}$ compared to the case of $\mathrm{sXT}=-25 \mathrm{~dB}$. This is explained by the extensive exchange of power between the

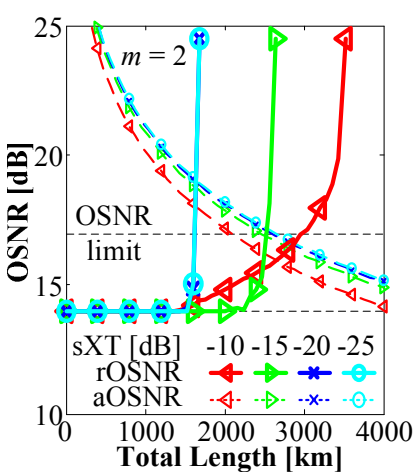

(a)

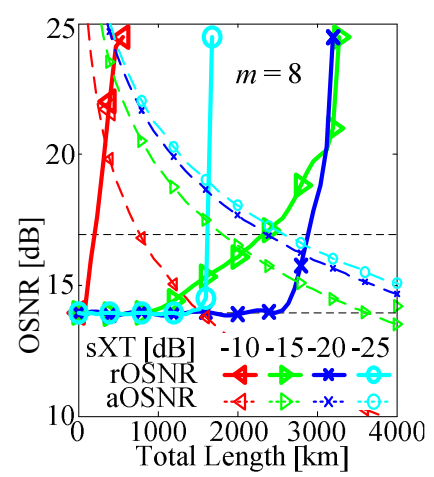

(b)
Fig. 4. Required OSNR and available OSNR for the ILD-FMF link as a function of total length, for: (a) $m=2$ and (b) $m=8$.

different modes in the splices, which introduces an averaging effect of the group velocities of the respective modes, thereby reducing the increase rate of the CIR length. Note that, this method is applicable independently of the number of modes.

The analysis up to now considered the introduction of sXT by splices in the absence of MDL. However, for significant MDL levels the DSP equalizer performance is degraded [9]. Since sXT, $\alpha_{S}$ and MDL increase simultaneously with the core displacement, see Fig. 1, there is an optimal sXT value ( $m$ dependent) for which the penalty due MDL equals the gain due to sXT. This is analyzed in Section III for MDM systems with 3 modes. For more than 3 modes, the optimum sXT and $m$ are expected to vary due to the MDL impact.

\section{Simulation Results}

\section{A. Inherently Low DMD FMF}

In order to understand the impact of the proposed method on the distance reach (DR) of the span configuration presented in Fig. 2(a), the rOSNR and aOSNR are analyzed first. Fig. 4 shows the OSNR results for $l D M D=9 \mathrm{ps} / \mathrm{km}$ and different $m$ values. In Fig. 4(a) and (b) it can be seen that, by increasing sXT, higher distances can be reached for the same required OSNR due to a reduction of the CIR spread, except for $m=8$ and $\mathrm{sXT}=-10 \mathrm{~dB}$. In the latter case, the performance degradation is justified by the increase of MDL, since this is the only source of penalty increasing from $m=2$ to $m=8$ and no penalty is visible for $m=2$ and $\mathrm{sXT}=-10 \mathrm{~dB}$. Additionally it can be seen that, by increasing $m$ and/or sXT, the aOSNR decreases, which is due to the increase of the $\alpha_{\mathrm{S}}$ and MDL per splice and/or number of splices per span. The DR is calculated from Fig. 4, taking into account the intersection of the rOSNR curves with the aOSNR curves or with the OSNR limit (defined as a the maximum OSNR penalty of $3 \mathrm{~dB}$ for back-toback).

Fig. 5 shows the DR as a function of (sXT, $\left.\alpha_{S}\right)$ given by Fig. 1, for different $I D M D$ values and different $m$ values. All curves in Fig. 5 can be decomposed in three different regions. In the first region (low sXT values), the DR does not vary with $m$ or sXT, which means that the splices do not have impact on the performance, for the evaluated cases. The DR is determined by the aOSNR $(\sim 13.95 \mathrm{~dB}$, at $\sim 5500 \mathrm{~km})$ for $l D M D \leq 3 \mathrm{ps} / \mathrm{km}$, and by the maximum CIR length (15 ns) for 


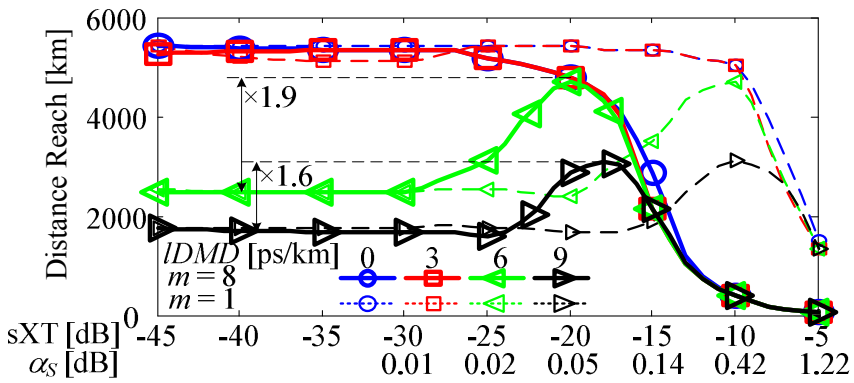

Fig. 5. Distance reach for the ILD-FMF link as a function of (sXT, $\left.\alpha_{S}\right)$.

$l D M D>3 \mathrm{ps} / \mathrm{km}$. For $l D M D>3 \mathrm{ps} / \mathrm{km}$, the $\mathrm{DR}$ is given by $15 \mathrm{~ns} / l D M D$ (meaning that the impact of $\mathrm{dXT}$ and $\mathrm{sXT}$ is negligible). In the second region (moderate sXT values), DR increases due to the sXT averaging effect (see Section II C). For $l D M D>3 \mathrm{ps} / \mathrm{km}$ there is an increase by a factor from 1.6 to 1.9 . However, for $l D M D \leq 3 \mathrm{ps} / \mathrm{km}$ there is no DR increase since the performance is determined by the aOSNR. Note that the sXT value corresponding to the maximum DR decreases with increasing $m$, since for a higher number of splices less sXT per splice is needed for the same CIR length reduction. In the third region (high sXT values), the DR decreases due to the high $\alpha_{S}$ and MDL values (see Section II C) for any $l D M D$ or $m$ value. In practice, some fluctuation of the sXT obtained through splicing is expectable. Further results have shown that, when considering sXT following a Gaussian distribution with a standard deviation of $0.5 \mathrm{~dB}$ (see Section II A), the distance gain remained nearly.

\section{B. DMD Compensated FMF}

In this sub-section the performance of the span configuration shown in Fig. 2 b) is analyzed. Fig. 6 shows the DR as a function of (sXT, $\alpha_{S}$ ) given by Fig. 1, for different $a D M D$ and $m$ values, calculated considering a methodology similar to the one used in Section III A. The DR results in Fig. 6 for $m=8$ are similar to the results in Fig. 5 for $m=8$, with the same limiting effects. The DR increase factors for $m=8$, are 1.9 for $a D M D=6 \mathrm{ps} / \mathrm{km}$ and 1.6 for $a D M D=9 \mathrm{ps} / \mathrm{km}$. However, for $m=1$, increasing sXT only leads to an increased CIR length and consequent shorter DR, independently of the $a D M D$ value. The difference of performance between $m=1$ and $m=8$ is present even for negligible sXT (particularly for $a D M D=3 \mathrm{ps} / \mathrm{km}$ ). This is explained due to the presence of dXT [4] and consequent exchange of a fraction of power between the modes for which there is an excess accumulation of DMD in each segment (in comparison to $N \times L \times a D M D$, when absence of XT is considered). For the fraction of power exchange around the middle of the segment, the accumulated $\mathrm{DMD}$ in one segment can reach $\pm[|p D M D| \times L p+$ $|n D M D| \times L n]$. For $m=1$, accumulated DMD can reach $16 \mathrm{~ns}$ in one segment, a value above the maximum CIR length. On the other hand, for $m=8$, the maximum accumulated DMD can reach only $2 \mathrm{~ns}$ in one segment. Therefore, for the DC-FMF case, $m$ should be high, even for perfect splices. Finally, it can be concluded that reducing the maximum accumulated DMD over one segment to a low fraction (around $13 \%$ ) of the compensation time interval provided by the DSP taps, the impact of $a D M D$ can be reduced by increasing the SXT as in the ILD-FMF case. Note that, concerning the

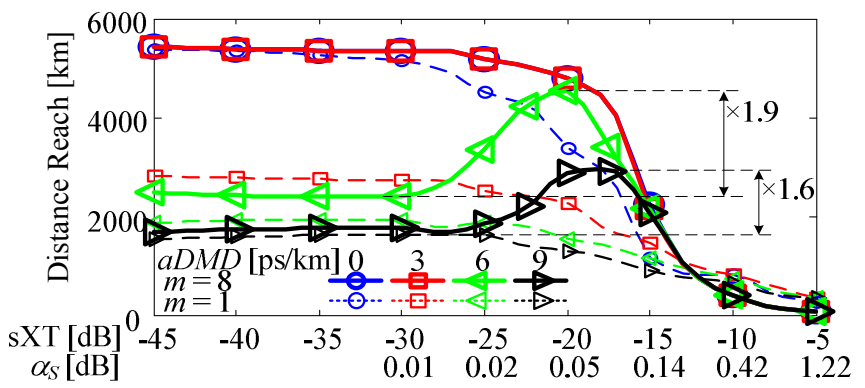

Fig. 6. Distance reach for the DC-FMF link as a function of (sXT, $\alpha_{S}$ ).

eventual sXT fluctuations in practice, the comments for Fig. 5 apply also for Fig. 6.

\section{CONCLUSION}

In this letter, we propose and prove that the introduction of fiber splices allows the improvement of the distance reach of MDM transmission systems. The concept was applied to ultralong-haul transmission over ILD-FMFs and DC-FMFs. The optimum number of segments/splices per span and respective $\mathrm{XT}$ values were determined taking into account the consequent MDL. The results show that the DR can be increased by a factor from 1.6 to 1.9 for the considered transmission systems. Additionally the results show that, for the case of DC-FMFs, the DR can be improved just by increasing $m$, even for negligible splices XT.

\section{REFERENCES}

[1] R. Ryf, et al., "Mode-Division Multiplexing Over $96 \mathrm{~km}$ of Few-Mode Fiber Using Coherent $6 \times 6$ MIMO Processing," J. Lightw. Technol, vol. 30, no. 4, pp. 521-531, 2012.

[2] K. Ho, J. Kahn, "Statistics of group delays in multimode fiber with strong mode coupling," J. Lightw. Technol, vol. 29, no. 21, pp. 31193128, 2011.

[3] S. Arik, D. Askarov, J. Kahn, "Effect of mode coupling on signal processing complexity in mode-division multiplexing," J. Lightw. Technol, vol. 31, no. 3, pp. 423-431, 2013.

[4] L. Gruner-Nielsen, Y. Sun, J. Nicholson, D. Jakobsen, R. Lingle, B. Palsdottir, "Few Mode Transmission Fiber with low DGD, low Mode Coupling and low Loss," in Proc. OFC'2012, USA, paper PDP5A.1.

[5] S. Warm, K. Petermann, "Splice loss requirements in multi-mode fiber mode-division-multiplex transmission links," OSA Opt. Express, vol. 21, no. 1, pp. 519-532, 2013.

[6] F. Ferreira, et al., "Nonlinear Semi-Analytical Model for Simulation of Few-Mode Fiber Transmission," IEEE Photon. Technol. Lett., vol. 24, no. 4, pp. 240-242, Feb. 15, 2012.

[7] P. Winzer, G. Foschini, "MIMO capacities and outage probabilities in spatially multiplexed optical transport systems," OSA Opt. Express, vol. 19, no. 17, pp. 16680-16696, 2011.

[8] F. Ferreira, D. Fonseca, H. Silva, "Design of Few Mode Fibers with Arbitrary and Flattened Differential Mode Delay," IEEE Photon. Technol. Lett., vol. 25, no. 5, pp. 438-441, 2013.

[9] A. Lobato, et al., "Impact of Mode Coupling on the Mode-Dependent Loss Tolerance in Few-Mode Fiber Transmission," OSA Opt. Express, vol. 20, pp. 29776-29783, 2012.

[10] R. Ryf, et al., "Low-Loss Mode Coupler for Mode-Multiplexed transmission in Few-Mode Fiber," in Proc. OFC'2012, USA, paper PDP5B.5.

[11] M. Li, et al., "Low delay and large effective area few-mode fibers for mode-division multiplexing," in Proc. OECC'2012, pp. 495-496, paper $5 \mathrm{C} 3-2$.

[12] S. Randel, et al., "Mode-Multiplexed $6 \times 20$-GBd QPSK Transmission over 1200-km DGD-Compensated Few-Mode Fiber," in Proc. OFC'2012, USA, paper PDP5C.5. 